Article

\title{
Knowledge-Based Recommendation for On-Demand Mapping: Application to Nautical Charts
}

\author{
Bilal Koteich ${ }^{1, * \mathbb{C}}$, Éric Saux ${ }^{1}$ (1) and Wissame Laddada ${ }^{2}$ \\ 1 Naval Academy Research Institute, BCRM Brest, CC 600, CEDEX 9, Lanvéoc-Poulmic, 29240 Brest, France; \\ eric.saux@ecole-navale.fr \\ 2 Laboratoire d'Informatique, du Traitement de l'Information et des Systèmes (LITIS), Univrsité de Rouen, \\ 76800 Saint-Étienne-du-Rouvray, France; wissame.laddada@univ-rouen.fr \\ * Correspondence: bilal.koteich@ecole-navale.fr
}

Citation: Koteich, B.; Saux, E.; Laddada W. Knowledge-Based Recommendation for On-Demand Mapping: Application to Nautical Charts. ISPRS Int. J. Geo-Inf. 2021, 10 786. https://doi.org/10.3390/ ijgi10110786

Academic Editor: Wolfgang Kainz

Received: 28 September 2021

Accepted: 12 November 2021

Published: 17 November 2021

Publisher's Note: MDPI stays neutral with regard to jurisdictional claims in published maps and institutional affiliations.

Copyright: (C) 2021 by the authors Licensee MDPI, Basel, Switzerland. This article is an open access article distributed under the terms and conditions of the Creative Commons Attribution (CC BY) license (https:// creativecommons.org/licenses/by/ $4.0 /)$.

\begin{abstract}
Maps have long been seen as a single cartographic product for different uses, with the user having to adapt their interpretation to his or her own needs. On-demand mapping reverses this paradigm in that it is the map that adapts to the user's needs and context of use. Still often manual and reserved for professionals, on-demand mapping is evolving toward an automation of its processes and a democratization of its use. An on-demand mapping service is a chain of several consecutive steps leading to a target map that precisely meets the needs and requirements of a user. This article addresses the issue of selecting relevant thematic layers with a specific context of use. We propose a knowledge-based recommendation approach that aims to guide a cartographer through the process of map-making. Our system is based on high- and low-level ontologies, the latter modeling the concepts specific to different types of maps targeted. By focusing on maritime maps, we address the representation of knowledge in this context of use, where recommendations rely on axiomatic and rule-based reasoning. For this purpose, we choose description logics as a formalism for knowledge representation in order to make cartographic knowledge machine readable.
\end{abstract}

Keywords: ontology; knowledge representation and reasoning; on-demand mapping; recommendation system; cartography

\section{Introduction}

The increase in the use of maps over the past few decades in everyday activities, accelerated by the digital production and dissemination of maps and the widespread availability of low-cost, location-sensitive devices, has made the work of cartographers and map display designers more challenging. Mapping agencies, such as the Ordnance Survey (OS) in the U.K., the National Institute of Geographic and Forest Information (IGN) or the Hydrographic and Oceanographic Service (SHOM) in France, have recognized for a long time this gap between the maps provided to the user and the maps that the user would need. This is one of the reasons why they offer on-demand mapping services. This type of service allows to meet precisely the requirements of a user and to ensure the production of a high-quality map. However, despite the scientific and technical progress, it is an expensive service because it requires qualified human resources.

In order to reduce the costs of producing personalized maps, geographic agencies have developed geographic web services (e.g., Ordnance Survey OnDemand Web Map Service (https: / / www.ordnancesurvey.co.uk/business-government/tools-support/ ondemand-map-service) (accessed on 28 September 2021), and Data SHOM Portal (https:/ / data.shom.fr/) (accessed on 28 September 2021) that allow a user to view and download his or her own maps, but independently of a particular need and context of use. The user builds his or her own cartographic representation by merging the thematic layers made available by the storage infrastructure with eventually his/her own. Moreover, the 
geographic web services should go beyond the simple proposal of viewing and downloading data. It would be useful to be able to benefit from geographic services that interfere with business logic and understand the specific needs of the user.

We therefore want to propose a recommendation approach for the on-demand map based on a context representation model adapted to the design of static and dynamic maps. The objective is to allow a user to obtain the knowledge he/she requires in the course of his/her activities and to obtain a representation of this knowledge in such a cartographic format as could be proposed by a cartographer or a web service. The design of such an on-demand map service is a multidisciplinary research field, whose goal is to develop mechanisms that are capable, without human assistance, of collecting a set of user requirements and interpreting them to build a personalized map.

Automatic map creation is a complex process that has attracted the interest of many cartographers, geographers and computer scientists. The automatic creation of a personalized map raises several scientific issues [1,2], ranging from data selection and map generalization problems to visualization. In this paper, we limit ourselves to the process of selecting thematic layers by a recommendation approach that responds to the needs and the activities of a particular user, without addressing visualization and generalization problems. For implementation issues, we have focused our case studies on selecting knowledge for the implementation of on-demand maps in a maritime context, but it can be derived to others (topographic, geological, tourism, etc.).

The rest of the paper is organized as follows. In Section 2, we present a literature review on the on-demand mapping process and context modeling in the cartographic domain and recommendation systems. In Section 3, we describe the research problem with our preferred orientations. Then, we present the implementation of the proposed solution in Section 4. Section 5 focuses on some use case scenarios, and finally, a discussion concludes this proposal in Section 6.

\section{Literature Review}

\subsection{On-Demand Mapping}

On-demand mapping is the research field that aims to automatically derive customized maps based on users' requirements. Many existing research works in the field of geographic information sciences (GIS) are related to on-demand mapping. According to Cecconi [3], on-demand mapping is defined as "the creation of a cartographic product upon a user request appropriate to its scale and purpose".

Figure 1 represents the main steps of an on-demand mapping system. These steps are necessary to derive, manually or automatically, a customized map. Each step listed above is a research field of its own [2].

Sarjakoski and Sarjakoski [4] implemented the first on-demand mapping prototype as part of the GiMoDig (2001-2004) project (Geospatial Info-Mobility Service by RealTime Data-Integration and Generalization (GiMoDig) project, IST-2000-30090, funded by the European Union through the Information Society Technologies (IST) program). The authors tried to improve the accessibility and interoperability of national topographic databases in a mobile context. The key techniques were data integration and real-time generalization. Custom map specifications were built from context parameters collected from the user and an internal knowledge base. Bucher et al. [5], at the COGIT laboratory of IGN France, specified a series of web services to provide on-demand maps based on users' specifications: a map specification service, a legend definition service, and a legend evaluation service. The first service helps the user in defining some of the abstract properties of their map. The two other services make use of the large knowledge base about legends to propose adequate symbolization. Foerster et al. [6] proposed a distributed architecture for on-demand web mapping by formalizing user requirements in UML and XML models. As a core of the architecture, a so-called generalization-enabled web map service is presented to automate the generalization process on the web. Gould [7] developed an on-demand mapping system based on an ontology for roads and road accidents. He 
aimed to model the process of generalization and devise a method for automatically selecting the appropriate algorithms for mapping geographic features at multiple scales, using an ontology. Balley et al. [8] worked on the translation of user requirements to map specifications. Map specifications rely on generalization, data production, data integration and legend design. The authors designed a map specifications model representing the principle of cartographic constraints to support not only generalization, but also other processes required by on-demand mapping, notably data integration. A use case of translation of user preferences to map specifications is shown by collecting user preferences in order to infer an appropriate map color and map legend.

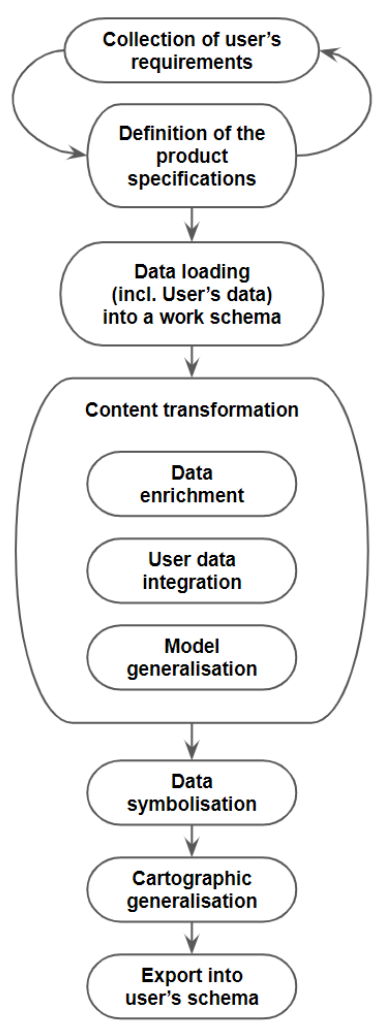

Figure 1. Main steps of the on-demand mapping process (extracted from [2]).

The state of the art points out that the existing research studies in the on-demand mapping domain mainly focus on user data integration, model and cartographic generalizations, data symbolization, definition of map specifications (designing map legends, map colors, etc.), etc. In our research, we address the problem of transforming users' requirements to map specifications (i.e., the first two initial steps in Figure 1), and more precisely, selecting relevant thematic data/layers according to the users' requirements and context.

\subsection{Contextual Cartography Modeling}

Before focusing more extensively on the modeling of the cartographic context, different works exist on context modeling from a general point of view. A commonly accepted definition of context was proposed by [9]. According to him, a context is defined as "any information that can be used to characterize the situation of an entity. An entity is a person, place, or object that is considered relevant to the interaction between a user and an application, including the user and applications themselves". In other words, a context is determined by the state of the values of the parameters relating to the characterization of a situation. It is a set of information that influences a task performed by a person or characterizes a specific situation in a computer system. A context-aware system is defined as follows: "A system is context-aware if it uses context to provide relevant information and/or services to 
the user, where relevancy depends on the user's task" [10]. Chen and Kotz [11] defined two classes of context-aware systems: active and passive systems. An active system is a system that takes into account the change of the dynamic contextual information and adapts its behavior according to the current situation, whereas a passive system is not able to update its behavior following a change of the context. Cabrera et al. [12] proposed a high-level categorization of context in order to build a contextual ontology that consists of defining a glossary of terms from the concepts corresponding to the first level of hierarchy of several proposed context models.

Strang et al. [13] presented a survey of six context modeling approaches: key-value models, markup scheme models, graphical models, object-oriented models, logic-based models and ontology-based models. Their analysis favors the ontology-based model for context modeling. According to Wang [14], the reasons for developing context models based on ontology rely on the following:

- Knowledge sharing: the use of context ontology enables computational entities, such as agents and services (e.g., in pervasive computing environments) to have a common set of concepts about context while interacting with one another.

- Logic inference: based on ontology, context-aware computing can exploit various existing logic reasoning mechanisms to deduce high-level conceptual context from lowlevel.

- Knowledge reuse: by reusing well-defined ontologies of different domains (e.g., temporal and spatial ontologies), we can compose large-scale context ontology without starting from scratch.

Focusing on the literature review on contextual cartography, several research works were conducted in order to introduce the notion of context in cartography, especially in mobile systems. First attempts to adapt visualization in mobile cartography were introduced by Reichenbacher [15] and Zipf [16]. Reichenbacher presented a conceptual framework for mobile cartography based on three essential components for visualization adaptation: the user, the context, and the task. The notion of context in digital mapping was later studied by Nivala and Sarjakoski [17] in their work on digital maps for mobile systems as part of the GiMoDig project (2001-2004). These researchers first relied on the definitions of context proposed by Schilit [18] and Dey [9]. Then, they proposed a context classification adapted to maps to describe a cartographic context in mobile systems based on five general context categories: computing, user, physical, time, and history. Each general context category includes a set of context categories for a mobile map as presented in Table 1.

In Section 4.1, we use the same approach as Cabrera's to define a high-level categorization, adapting their categorization to the cartographic domain with Nivala and Sarjakoski's classification [17] and integrating Chen and Kotz's active/passive approach [11]. 
Table 1. Categorization of contexts and their characteristics for mobile cartographic services (extracted from [17]).

\begin{tabular}{|c|c|c|}
\hline $\begin{array}{l}\text { General Context } \\
\text { Categories }\end{array}$ & $\begin{array}{l}\text { Context Categories for } \\
\text { Mobile Map }\end{array}$ & Features \\
\hline Computing & System & $\begin{array}{l}\text { Size of display } \\
\text { Type of display (color etc.) } \\
\text { Input method (touch panel, buttons) } \\
\text { Network connectivity } \\
\text { Communication costs and bandwidth } \\
\text { Nearby resources(printers, displays) }\end{array}$ \\
\hline User & $\begin{array}{l}\text { Purpose of use } \\
\text { User } \\
\text { Social } \\
\text { Cultural }\end{array}$ & $\begin{array}{l}\text { User's tasks } \\
\text { User's profile (experience etc.) } \\
\text { People nearby } \\
\text { Characters, date and time format }\end{array}$ \\
\hline Physical & $\begin{array}{l}\text { Physical surroundings } \\
\text { Location } \\
\text { Orientation }\end{array}$ & $\begin{array}{l}\text { Lighting, temperature, weather conditions, etc. } \\
\text { Surrounding landscape } \\
\text { User's direction of movement }\end{array}$ \\
\hline Time & Time & $\begin{array}{l}\text { Time of day } \\
\text { Week, month } \\
\text { Season of the year }\end{array}$ \\
\hline History & Navigation history & $\begin{array}{l}\text { Previous locations } \\
\text { Former requirements and points of interest }\end{array}$ \\
\hline
\end{tabular}

\subsection{Recommendation Systems}

Recommendation systems are tools for interacting with large and complex information systems. The goal of these systems is to provide to a user a personalized view of these information systems by prioritizing relevant resources based on their preferences in order to assist him/her in different decision-making processes.

According to the literature [19], widely used recommendation approaches are content based, collaborative filtering and knowledge based. Collaborative filtering $(\mathrm{CF})$ approaches are based on the opinion of a group of users who have the same preferences-ratings of items by a community of users-to generate recommendations. Collaborative filtering algorithms have the advantage of using only historical data; no knowledge of the items is required. However, they suffer from a "cold-start" problem; a new user cannot receive any recommendations before rating several items, and a new item cannot be recommended before being rated by a number of users [20]. Content-based filtering (CB) approaches use item features to recommend items similar to those in which the user has expressed interest. CB has no cold start problem, but is unable to provide the serendipitous (serendipity is the luck some people have in finding or creating interesting or valuable things by chance (Collins COBUILD Advanced Dictionary)) recommendations that CF generates. Lastly, knowledge-based approaches (KB) use domain knowledge in a structured form to produce personalized recommendations. KB approaches avoid the cold start problem and have the advantage of enhanced reliability, as the background knowledge is free of noise. However, knowledge-based systems require considerable knowledge acquisition effort for setup and maintenance during their lifetime [21], which makes them more expensive to develop and maintain.

\section{Problem Statement and Preferred Orientations}

The application objective is to develop a system that aims to assist a cartographer in the process of creating an on-demand map, to select the relevant thematic layers according to user requirements and a given context of use. According to the main steps of the on-demand mapping process presented in Figure 1, we focus exclusively on the definition of thematic layers, according to user requirements (i.e., "Definition of the product specifications" step). In order to make a machine able to transmit and infer the cartographic knowledge adapted 
to a given context derived from user requirements, the machine must be able to understand the knowledge (information) that it handles. This is a step toward the automation of cartographic systems. To make map information machine readable, it is necessary to model and represent this information. This requires the use of a representation formalism with a defined syntax and formal semantics. The most suitable formalism for knowledge representation is description logic (DL). DL is known as the reference for the creation of ontologies. DL allows us to formalize simple or complex concepts in a hierarchical way: the properties-roles - that link the concepts and individuals. This formalism is supported by languages, such as OWL (Web Ontology Language), that allow the implementation of formalized ontologies and also have reasoners for inference, such as Pellet, FacT++, or Hermit, taking into account the temporal and spatial dimensions.

One solution for building such systems is the recommendation approach. According to Pathak et al. [22], recommendation systems have proved their ability to improve the decision-making process. In our research, we choose the knowledge-based approach for different reasons. The advantages of this approach can be summarized as follows:

- No cold start problem: the recommendation system can start producing recommendations for new users without the need of rating any item before.

- $\quad$ Assured quality: since the knowledge-based recommendation systems try to match between the user's requirements/preferences and the items, the results of the recommendation are accurate and deterministic.

- Criticality of the domain: according to Ramezani et al. [20], the cost of a wrong recommendation must be considered. In critical domains, a knowledge-based approach is needed, as a correct and explainable recommendation is impossible with other approaches.

In order to make the knowledge-based system sensitive to the context for the ondemand mapping process, we need to couple the research on the knowledge-based recommendation systems and the contextual cartographic modeling presented respectively in Sections 2.2 and 2.3. In order to reach this target, we propose to make recommendations based on an axiomatic and rule-based formalization, taking into account the different dimensions involved in the cartographic context, including spatial and temporal dimensions. In the following section, we present a methodology to develop such an ontology of contextual cartography that will be the basis of the recommendation system.

\section{Methodology}

The first step toward context modeling and representation for an on-demand mapping is the conceptualization. This step consists in categorizing the objects of the real world into abstract concepts. Once the concepts are defined, we use description logic as a formalism for knowledge representation in order to represent the semantics of concepts in a structured way and then extract implicit knowledge by ontological reasoning. In order to create our knowledge base, we implement the concepts as an ontological model using Protégé (https://protege.stanford.edu/) (accessed on 28 September 2021) with a set of SWRL (Semantic Web Rule Language) rules for rule-based reasoning. Lastly, we instantiate the model in order to illustrate a concrete use case to infer relevant thematic layers according to a given context of use. Although the methodology is general for the on-demand map, we focus on the on-demand map in the maritime domain to concretize and illustrate the proposed approach.

\subsection{Conceptualization}

In their work on a contextual ontology for the recommendation of services, Cabrera et al. [12] proposed a high-level conceptualization of context dimensions, including time, profile, location, activity, environment, etc. Each defined concept is a sub-class of the concept context information. In our conceptualization, we adapt this approach to the cartographic domain, taking into account both the context dimensions defined by Nivala and Sarjakoski [17] (see Figure 1) and the passive/active approach of Chen and Kotz [11]. 
In a manual process of on-demand mapping, the cartographer defines the map specifications (i.e., the relevant data to be mapped), according to the user's profile, the purpose of use, the geographical area, etc. The automation of this process requires additional knowledge or concepts, such as the user's expertise (e.g., expert/non-expert), user's community (e.g., surfing club), and the policies and restrictions of the practice area (e.g., caution area).

Toward an automated process of on-demand mapping, a user requesting a map has a profile, plans for an activity, and may also belong to a community. The activity takes place in a practice area (i.e., location), has a temporal state (i.e., time) and is surrounded by a physical environment. Based on the domain of application, the physical environment, a subclass of the environment, represents the environmental conditions: weather conditions, traffic conditions, oceanographic forecast, etc. The physical environment may be exposed to events, and the practice area might have some policies and restrictions. Context information is defined by any information describing a user's profile/preferences (e.g., profile and activity) or the surrounding environment (e.g., event and time). We have divided context information into two classes: static context information and dynamic context information to make the model useful both for static maps and adaptive maps (i.e., navigation systems that periodically adapt their display according to a given context). Static context information is information that persists throughout a long time (i.e., during a session of use of the system). For instance, the user's profile or user's activity are static context information since they do not change during the recommendation process. Dynamic context information is information that may have changes over a short time, maybe several times during a single recommendation session. For instance, traffic information for road maps is dynamic context information since this information changes during the same recommendation. One or more pieces of context information provide a defined context.

In order to make the model more generic, we have defined high-level concepts as a first step as shown in Table 2. Each high-level concept includes a set of low-level concepts describing a context in a specific domain of application (e.g., weather, population density, navigation, and tourism maps).

Table 2. High-level concepts for context description.

\begin{tabular}{|c|c|}
\hline High-Level Concept & Description \\
\hline Context & $\begin{array}{l}\text { A collection of values extracted from } \\
\text { context information }\end{array}$ \\
\hline Context Information & $\begin{array}{l}\text { Any information that can be used to describe } \\
\text { user's profile or the surrounding environment }\end{array}$ \\
\hline $\begin{array}{l}\text { Static Context } \\
\text { Information }\end{array}$ & $\begin{array}{c}\text { A context information that persists } \\
\text { during the same recommendation session } \\
\text { (e.g., user's profile) }\end{array}$ \\
\hline $\begin{array}{l}\text { Dynamic Context } \\
\text { Information }\end{array}$ & $\begin{array}{l}\text { A context information that changes } \\
\text { during the same recommendation session } \\
\text { (e.g., location) }\end{array}$ \\
\hline Situation & $\begin{array}{l}\text { A set of values extracted from dynamic } \\
\text { context information during a short time }\end{array}$ \\
\hline Activity & The purpose of use of the user (e.g., navigation) \\
\hline Time & The time during which the activity takes place \\
\hline Location & The area where the activity takes place \\
\hline Environment & Surrounding physical and computational environments \\
\hline Event & $\begin{array}{c}\text { It might be natural events (e.g., storm, rain and fire) } \\
\text { or human events (e.g., collision) }\end{array}$ \\
\hline Policy & $\begin{array}{c}\text { Regulations applied to a geographical area } \\
\text { (e.g., caution area) }\end{array}$ \\
\hline User & The end-user of the map \\
\hline Profile & The user's profile (e.g., profession, expertise and community) \\
\hline
\end{tabular}

In the following, we decide to focus on the definition of low-level relevant concepts in the maritime domain that affect the process of on-demand nautical map making. To do this, 
we extracted some knowledge related to the maritime environment and navigation from reference books [23-25], as well from the SHOM (https: / / data.shom.fr/) website (accessed on 28 September 2021). In addition, we also had discussions with experts in maritime navigation training from the French Naval Academy.

In order to illustrate some recommendation examples in the maritime domain, we have chosen to conceptualize some knowledge that will be used in the following to illustrate the usability of our approach to make recommendations. In a maritime domain, we considered that the physical environment consists of weather conditions, oceanographic forecast, tide conditions, etc. We defined the concept of visibility distance as a subclass of weather conditions. By definition, the visibility is the distance (in miles) at which an object can be clearly distinguished. The visibility distance is a class that determines the value of the visibility (in miles). Based on this value, we defined the class visibility situation, as a subclass of situation in order to represent the different visibility conditions. The visibility situation consists of good visibility, restricted visibility and bad visibility.

In our model, a situation implies a context. We have defined a set of contexts related to visibility situations as follows: good visibility context, restricted visibility context and bad visibility context. Other contexts are defined based on the user's activity, such as navigation context, fishing context, sailing context, etc. In a maritime environment, an event could be a natural event (e.g., intense fire) or human event (e.g., collision). Policies could be regulation (e.g., restricted area and fishery zone) or sovereignty (e.g., contiguous zone and exclusive economic zone). Table 3 summarizes some domain concepts for on-demand nautical map.

Table 3. Some domain concepts describing a context of on-demand nautical map.

\begin{tabular}{cc}
\hline High-Level Concept & Domain Concept \\
\hline Context & Fishing Context, Navigation Context, Surfing Context, etc. \\
Situation & Bad Visibility, Restricted Visibility, Good Visibility \\
Activity & Navigation, Transportation, Fishing, etc. \\
Time & Daytime, Nighttime \\
Location & Practice Area \\
Physical Environment & Weather Conditions, Tide Conditions, etc. \\
Event & Storm, Intense Fire, Collision, etc. \\
Policy & Regulation, Sovereignty \\
\hline
\end{tabular}

\subsection{Formalization}

Description logics [26] are a class of knowledge representation formalisms, which can be used to represent the knowledge of an application domain in a structured and formally well-understood way. In DLs, we formalize the relevant notions of an application domain by concept descriptions. A concept description is an expression built from atomic concepts, which are unary predicates, and atomic roles, or binary predicates, by using logical constructors and quantifiers provided by the particular DL language in use. In the following, we define some concepts using DL that we use in Section 5 in order to illustrate concrete scenarios. We restrict hereinafter to the concept definitions leading to different contexts, according to either a situation (e.g., a visibility situation) or an activity (e.g., fishing).

As presented in Section 4.1, we identify three visibility situations. According to [24], a bad visibility situation takes place when the visibility distance is less than 2 miles, or when the activity takes place at night. The restricted visibility takes place when the visibility distance is between 2 miles and 5 miles, and greater than 5 miles for good visibility. The concept of nighttime is a subclass of time, and it indicates the time between evening and morning: the time of darkness.

$$
\begin{gathered}
\text { NightTime } \sqsubseteq \text { Time } \\
\text { VisibilityDistance } \sqsubseteq \text { WeatherConditions } \\
\text { VisibilitySituation } \sqsubseteq \text { Situation }
\end{gathered}
$$


VisibilitySituation $\equiv$ GoodVisibility $\sqcup$ RestrictedVisibility $\sqcup$ BadVisibility

GoodVisibility $\equiv$ Situation $\sqcap \exists$ causedBy • (VisibilityDistance $\square$

$\exists$ hasVisibilityDistance. $(>5))$

RestrictedVisibility $\equiv$ Situation $\sqcap \exists$ causedBy $\cdot($ VisibilityDistance $\square$

$\exists$ hasVisibilityDistance.$(\geq 2) \sqcap \exists$ hasVisibilityDistance.$(\leq 5))$

BadVisibility $\equiv$ Situation $\sqcap \exists$ causedBy.$($ VisibilityDistance $\square$

$$
\exists \text { hasVisibilityDistance } .(<2) \sqcup(\exists \text { caused By } \text {. NightTime })
$$

$$
\text { BadVisibilityContext } \equiv \text { Context } \sqcap \exists \text { generatedBy . BadVisibility }
$$

According to a user's expertise, a user may be professional or standard. We define a professional user as a user whose expertise is equal to the predefined value "high", and a standard user is a user whose expertise is equal to "low" or "medium".

$$
\begin{gathered}
\text { ProfessionalUser } \equiv \text { User } \sqcap \exists \text { has Expertise } \cdot\{\text { high }\} \\
\text { StandardUser } \equiv \text { User } \sqcap(\exists \text { hasExpertise },\{\text { low }\} \sqcup \exists \text { has Expertise } .\{\text { medium }\})
\end{gathered}
$$

Le Guyader [27] presents a classification of human activities in the coastal maritime area. In this classification, we have the fishing concept that designates a professional fishing activity, and the casual and pleasure fishing concept related to a leisure activity. In order to define contexts related to fishing activities, we have relied on two types of context information: the activity and the user's expertise. We defined the concept of fishing context with two sub-contexts: the professional fishing context and the leisure fishing context. The professional fishing context indicates a fishing activity carried out by a professional user. The leisure fishing context takes place when a standard user is engaged in a fishing activity. We have the same principle with the sailing activity.

$$
\begin{gathered}
\text { Fishing } \sqsubseteq \text { Activity } \\
\text { Sailing } \sqsubseteq \text { Activity } \\
\text { FishingContext } \sqsubseteq \text { Context } \\
\text { SailingContext } \sqsubseteq \text { Context }
\end{gathered}
$$

ProfessionalFishingContext $\equiv$ Context $\sqcap \exists i$ STheContextOf $\cdot$ (Fishing $\square$

$$
\exists \text { has Actor. Professionaluser) }
$$

$$
\begin{aligned}
\text { LeisureFishingContext } \equiv \text { Context } \sqcap \exists \text { isTheContextOf } & \text { ( Fishing } \square \\
& \exists \text { has Actor. StandardUser })
\end{aligned}
$$

ProfessionalSailingContext $\equiv$ Context $\sqcap$ $\exists i s$ TheContextOf. (Sailing $\square$

$$
\exists \text { has Actor. Professionaluser) }
$$




$$
\begin{aligned}
\text { LeisureSailingContext } \equiv \text { Context } \sqcap \exists \text { isTheContextOf } & (\text { Sailing } \square \\
& \exists \text { has Actor. StandardUser })
\end{aligned}
$$

\subsection{Ontology Implementation}

Based on the concept formalization of Section 4.2, the conceptualization defined in Section 4.1 was implemented as an ontological model using Protégé. This implementation provides support for description logics reasoning. The high-level concepts were implemented as classes in order to obtain a high-level ontology (Figure 2). Then, we implemented the domain concepts as subclasses of the main concepts. Reusing existing ontologies is a crucial step in ontology development. It provides a useful starting point to be fully or partially reused. For instance, we used the GeoSPARQL (http: / / www.opengis.net/ont/geosparql\#) (accessed on 28 September 2021) standard ontology to represent the spatial dimension, and the OWL-Time ontology [28] to represent the temporal dimension. The FOAF (http:/ / xmlns.com/foaf/spec/) (accessed on 28 September 2021) ontology is used to represent the user's profile. The classification of maritime activities presented by Le Guyader [27] was integrated into our model as classes and subclasses. We also reused the ontological approach proposed by Tsatcha [29] to model the S-57 (http:/ / www.s-57.com/) (accessed on 28 September 2021) standard format. The S-57 model classifies hydrographic information (i.e., thematic layers) used for nautical charts making. In addition to hydrographic information, we have extracted two meteorological layers from the SHOM (https://data.shom.fr/) geoportal: oceanographic forecast and coastal observations. These thematic layers will be useful for the following use cases. All the layers (i.e., S-57 and oceanographic forecast) are subclasses of the class resources.

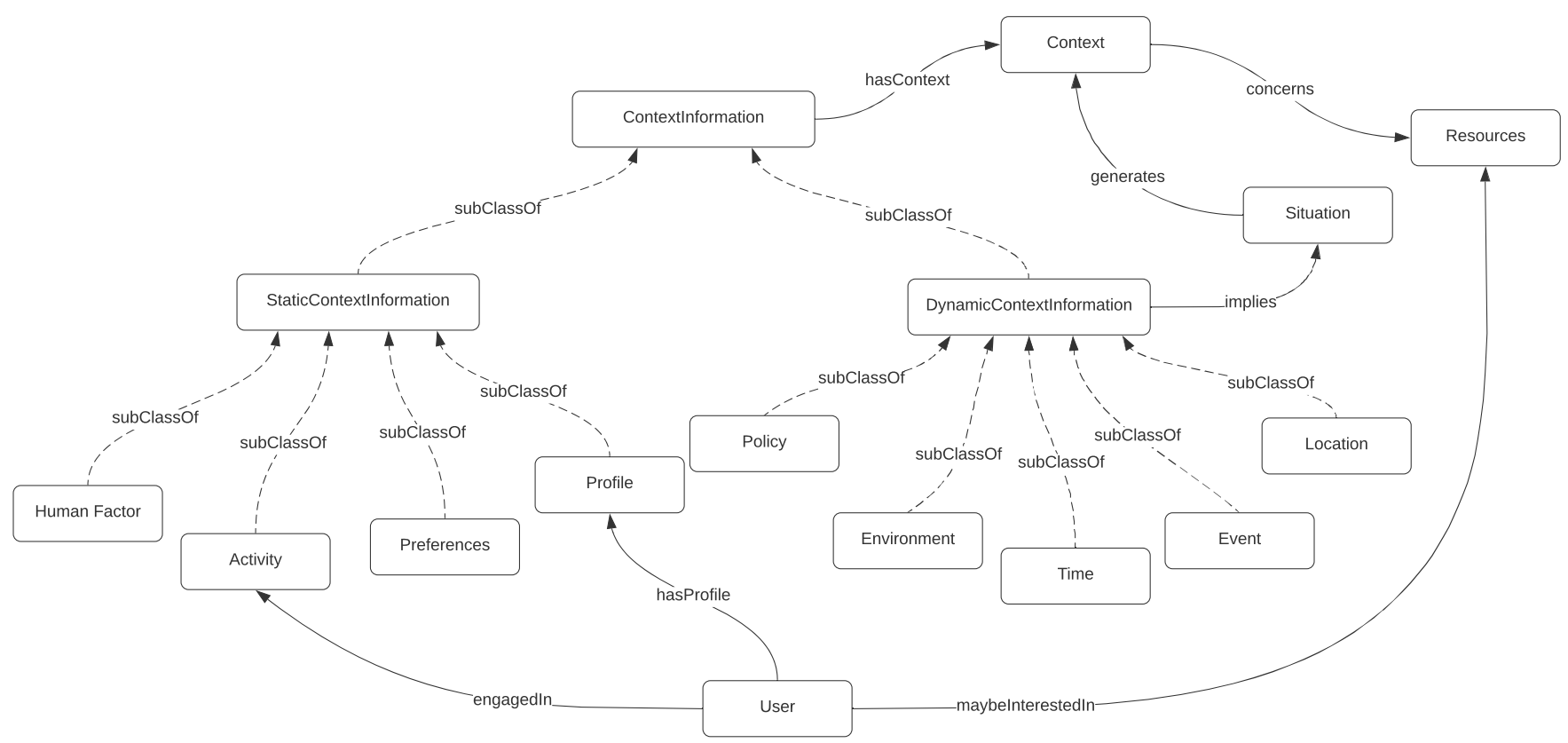

Figure 2. Overview of the proposed upper ontology.

The ontology we provide in this work consists of a set of sub-ontologies describing abstract concepts for on-demand maps. Thereafter, we extend these sub-ontologies by concepts related to a particular domain: on-demand maritime maps. Figures 3 and 4 depict the resulting ontologies, including their general relationships. In the following, we detail the sub-ontologies with their relationships (Table 4). 


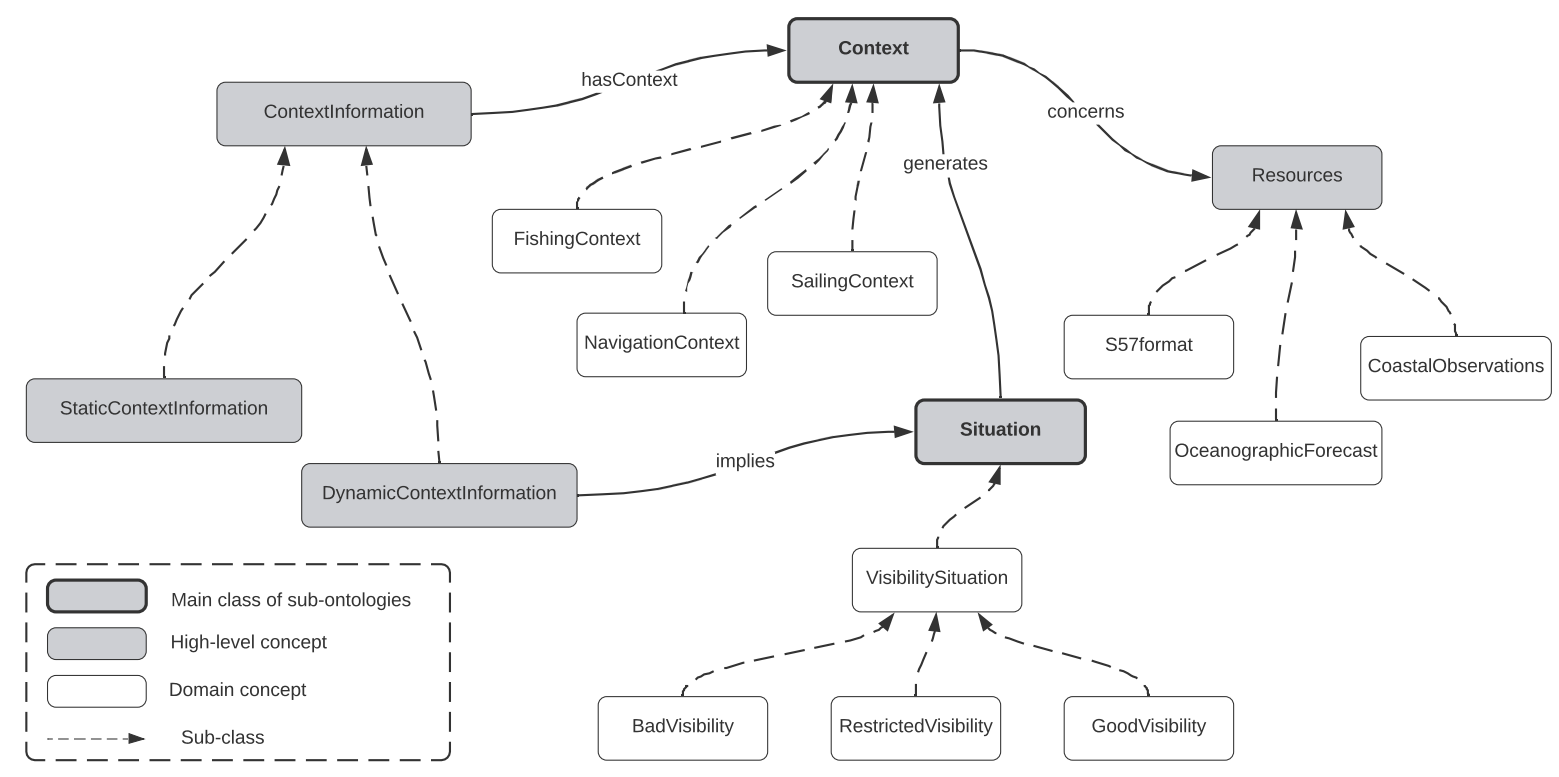

Figure 3. Partial taxonomy overview of concepts related to the sub-ontologies: context and situation.

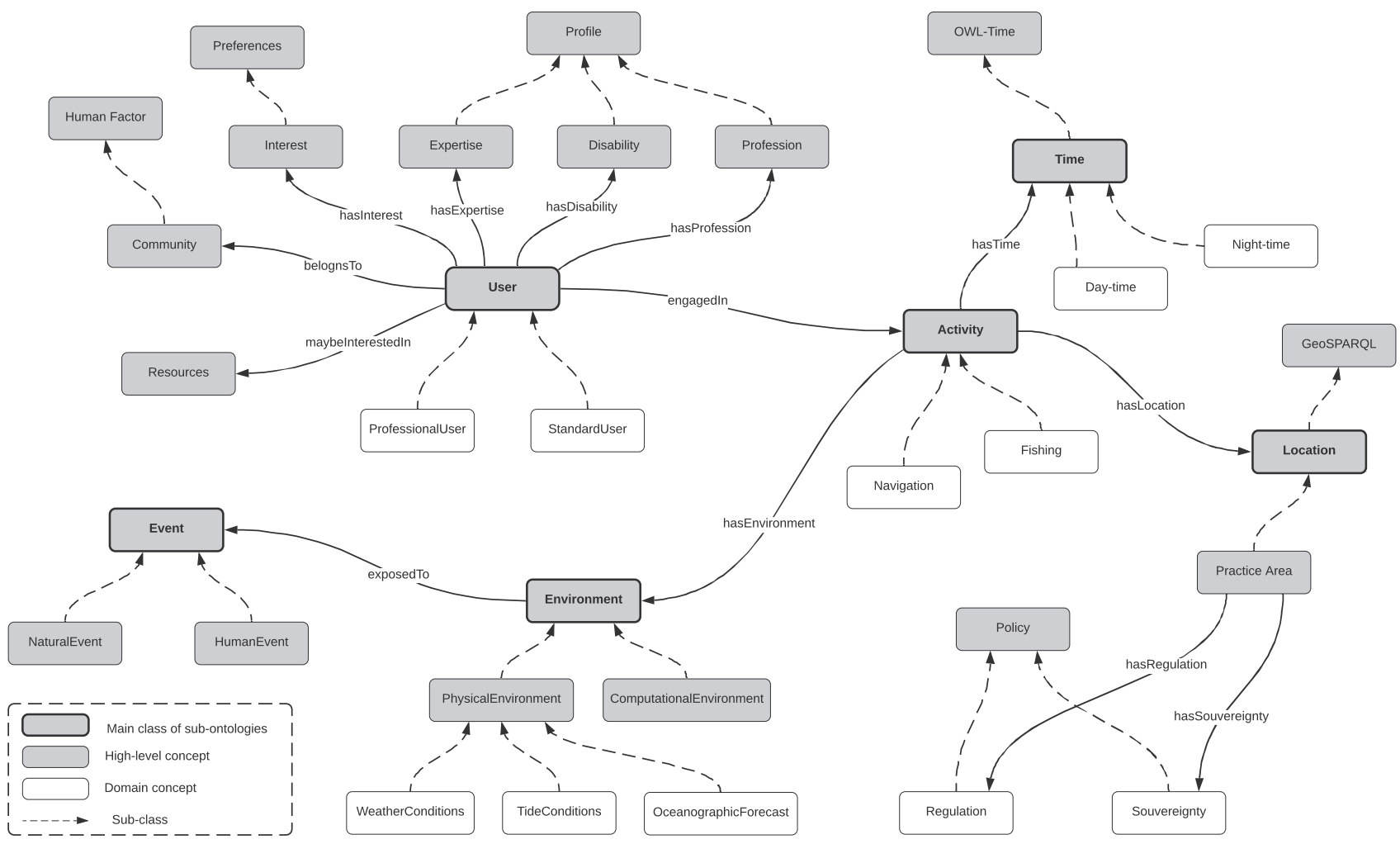

Figure 4. Partial taxonomy overview of concepts related to the sub-ontologies: event, user, activity, location, time, and environment. 
Table 4. Object properties between main classes.

\begin{tabular}{|c|c|c|}
\hline Object Property & Domain Class & Range Class \\
\hline engagedIn & User & Activity \\
\hline hasActor (三engagedIn ${ }^{-1}$ ) & Activity & User \\
\hline hasExpertise & User & Expertise \\
\hline maybeInterestedIn & User & Resources \\
\hline hasEnvironment & Activity & Environment \\
\hline hasTime & Activity & Time \\
\hline LocatedIn & Activity & Location \\
\hline hasContext & ContextInformation & Context \\
\hline $\begin{array}{l}\text { isTheContextOf } \\
\left(\equiv \text { hasContext }^{-1}\right)\end{array}$ & Context & ContextInformation \\
\hline exposedTo & PhysicalEnvironment & Event \\
\hline implies & DynamicContextInformation & Situation \\
\hline causedBy $\left(\equiv\right.$ implies $\left.^{-1}\right)$ & Situation & DynamicContextInformation \\
\hline generates & Situation & Context \\
\hline 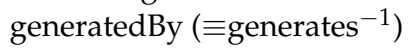 & Context & Situation \\
\hline concerns & Context & Resources \\
\hline hasConditions & PhysicalEnvironment & WeatherConditions \\
\hline
\end{tabular}

\subsubsection{User Ontology}

The user ontology consists of two main branches: the user's profile and his/her activity. On the one hand, the user's profile has an influence on the map-making process: it includes the profession of the user, the community to which he/she belongs, his/her expertise, disability and interests. Some of these factors affect the relevant data to be mapped (e.g., expertise and interest), and others affect the semiology of graphics in the maps (i.e., graphic techniques including shape, orientation, color and texture). For example, certain disabilities (e.g., a color-blind user) will directly affect the graphic semiology. On the other hand, the user is engaged in an activity. The activity is a crucial factor to infer relevant thematic layers (Figure 4).

\subsubsection{Activity Ontology}

Identifying the activity of the user is the most important stage in order to select the relevant thematic layers in the context of use related to it. An activity has a temporal dimension, either qualitative (e.g., day /night) or quantitative using the OWL-time ontology. An activity is located in a practice area, the area where the user is planning to carry out his/her activity. The practice area may have some restrictions, such as regulations (e.g., caution area and fishery zone) or sovereignty (e.g., contiguous zone and exclusive economic zone). The activity is also associated to a surrounding physical environment (Figure 4).

\subsubsection{Environment Ontology}

Environmental factors have a potential influence on the map display. This concept consists of two types: physical environment and computational environment. On the one hand, the computational environment describes the device used by the end-user (e.g., network connectivity and size of output display). These factors are related to the visual representation of the map (e.g., semiology and cartographic generalization). On the other hand, the physical environment has an impact on the process of selecting relevant thematic layers. For example, according to the weather conditions, the map may have different layers in different contexts of use. We defined the weather conditions as one of the physical environment factors (Figure 4).

\subsubsection{Location Ontology}

In order to take into consideration the spatial dimension, we used the GeoSPARQL ontology standard. The spatial dimension is limited to the user location, the geographical 
area where his/her activity takes place and the geographical coordinates of cartographic entities, which instantiate the thematic layers (Figure 4).

\subsubsection{Time Ontology}

The temporal dimension consists of two types: qualitative and quantitative. The OWL-time ontology is used to represent the quantitative time. Furthermore, the qualitative time could be represented with concepts such as daytime, nighttime, etc. (Figure 4).

\subsubsection{Event Ontology}

This ontology is limited to a set of predefined events that can occur during the user's activity. There are two types of events: human events, such as boat collisions or regatta, and natural events (e.g., intense fire and storm) (Figure 4).

\subsubsection{Context Ontology}

It is the most important part of the ontology. The class context is a generic concept from which we can define a set of contexts related to different application domains of on-demand maps (e.g., maritime cartography or land cartography). For implementing our case studies, we have defined a set of contexts related to maritime cartography. One or more pieces of context information form a context of use. Each defined context is associated to a set of relevant thematic layers using the object property "concerns" (Figure 3).

\subsubsection{Situation Ontology}

The situation ontology represents the state of the system during a short time. The state is derived from dynamic context information, which can change their values over a short time (i.e., during a recommendation session). A situation could be danger, a capacity for visibility, etc. Each situation generates a defined context. In the following case studies, we defined some situations related to visibility states (bad, restricted, or good visibility situations) varying according to the weather and time conditions (Figure 3).

\subsection{Reasoning}

The proposed approach aims to recommend to a user the relevant thematic layers, according to a given context of use. The reasoning process is the core of such recommendations. It consists of two ontological reasoning types: axiomatic reasoning and rule-based reasoning. Axioms are used to represent real-world knowledge in the ontologies, using the OWL syntax, while complex problems need additional description techniques. Our initial ontology formalization (Section 4.2) was extended with a defined rule base. These rules are formalized, using the Semantic Web Rule Language (SWRL) to express the required statements. SWRL is an expert-level solution or an adaptation for rule-based systems in the semantic web domain. Note that in order to preserve decidability in the reasoning process, SWRL rules are DL-safe rules (i.e., they can only be applied explicitly to existing individuals in the knowledge base and not to language components).

The axiomatic reasoning process aims to infer implicit knowledge from a set of asserted facts and axioms (see Section 4.2). We use ontological reasoning to infer the appropriate contexts of the user, the situations that take place during a session of use, and the user's class (i.e., professional or standard). On one side, a context is defined based on a set of asserted context information or based on a defined situation. On the other side, the situations may be inferred based on dynamic context information. Each situation generates a defined context. As a result, knowing the user's profile, the activity and the surrounding environment, one can deduce the context(s) of use in which the user is involved. Each defined context is associated to a set of thematic layers. The following example (Listing 1) shows how a context is associated to some relevant thematic layers, using the Manchester OWL syntax (https:/ / www.w3.org/2007/OWL/draft/ED-owl2-manchester-syntax-20081128/) (accessed on 28 September 2021): 
Listing 1: An example illustrating a class Context1 defined as a restriction on the data property concerns whose values are associated with the relevant thematic layers for the class.

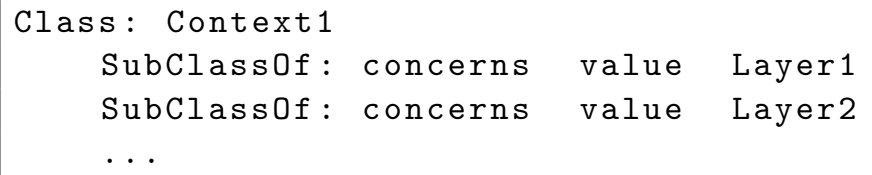

In addition to axiomatic reasoning, the rule-based reasoning process consists of inferring relevant thematic layers to the user's needs. Once the context(s) are inferred, we apply SWRL rules to infer the relation "maybeInterestedIn" between a user and some appropriate thematic layers. In the following, we present an example of three rules used in the reasoning process:

$$
\begin{array}{r}
\text { Rule } 1: \text { User }(? u) \wedge \text { engaged In }(? u, ? a) \wedge \text { hasContext }(? a, ? c) \\
\rightarrow \text { concerns }(? c, ? e) \\
\rightarrow \text { maybeInterested } \operatorname{In}(? u, ? e)
\end{array}
$$

Rule 2 : User $(? u) \wedge$ engagedIn $(? u, ? a) \wedge$ hasTime $(? a, ? t) \wedge$ implies $(? t, ? s)$ $\wedge$ generates $(? s, ? c) \wedge$ concerns $(? c, ? e) \rightarrow$ maybeInterested $\operatorname{In}(? u, ? e)$

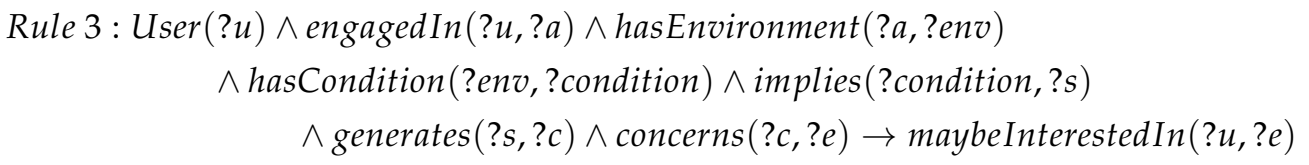

The first rule infers the thematic layers provided by a context related to some activities. The second one deals with inference related to qualitative temporal dimension. Finally, the third one provides recommendations based on environmental conditions.

\subsection{Architecture Framework}

Figure 5 presents an overview of the proposed recommendation system. This system is developed in Java programming language using OWL-API (https:/ / github.com/owlcs / owlapi/) (accessed on 28 September 2021), a Java library to deal with ontologies. The instantiation by assertion of the different classes and properties are realized in different ways: directly from the imported ontologies (e.g., the thematic layers from the ontology proposed by Tsatcha [29] to model the S-57 standard format), manually (e.g., for the classification proposed by Le Guyader [27] for the human activities in the coastal maritime area) or using an interface. For the latter case, the end-user enters his or her own data through a graphical user interface. This interface is a Java application developed by Gatin and De Montaignac [30] as part of the prototype for the recommendation of the thematic layers. It allows a user to enter his or her profile, activity, profession, expertise, etc., and store them in the knowledge base. In this base, axioms and rules are defined directly by the expert engineer in knowledge representation from the knowledge of the expert cartographer.

Once the required information is stored into the ontology, we apply the reasoning process. The results are a set of thematic layers relevant in some inferred context of use that are recommended to the cartographer to produce the on-demand map and indirectly to the user. The rule base was developed with the assistance of expert cartographers in order to select which thematic layers are relevant for each defined context. To go one step beyond the thematic layers selection, we converted the data of some layers of some electronic navigational charts (ENCs) from shapefile to RDF formats. The resulting triples are a set of cartographic entities with spatial coordinates, giving the possibility to make spatial inferences (see Section 6). 


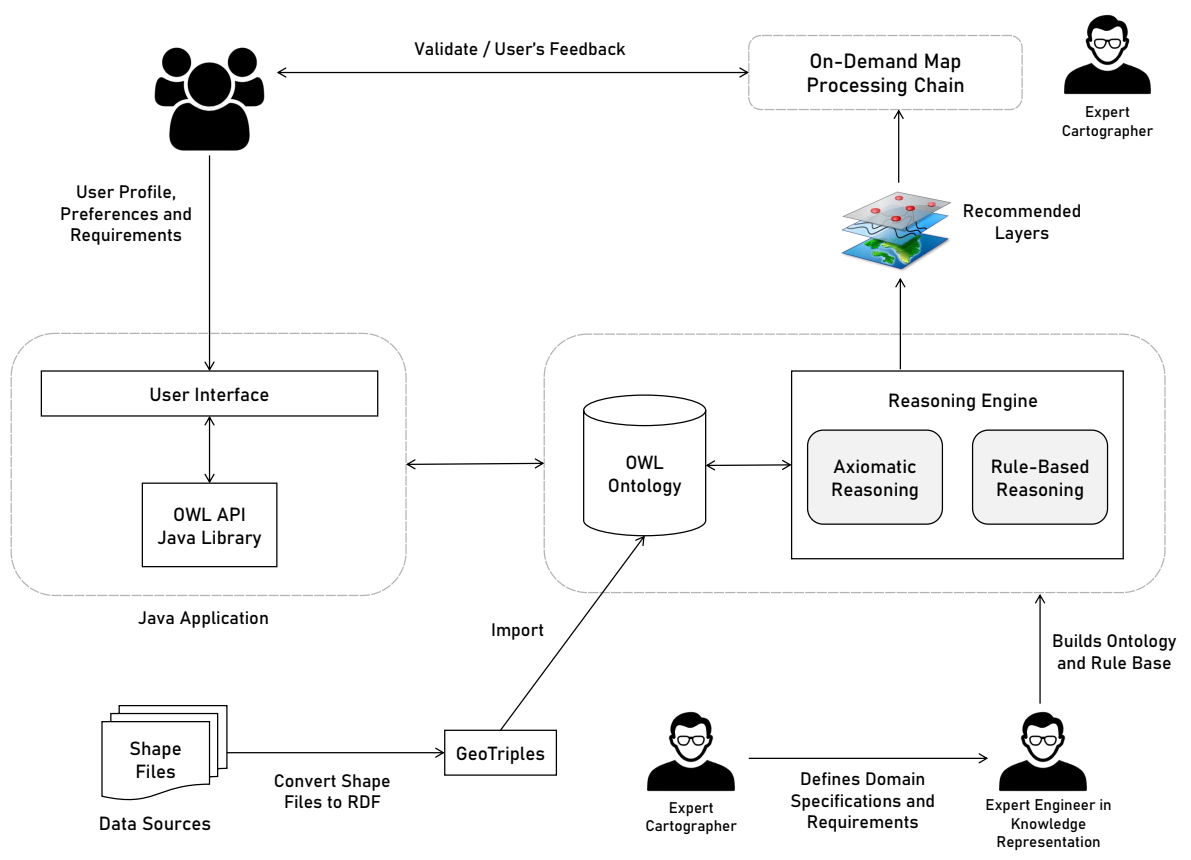

Figure 5. Architecture framework for the recommendation of thematic layers.

\section{Use Case Scenarios}

In this section, we present two use case scenarios for the recommendation of themes for an on-demand nautical chart. For each scenario, we present a table showing the instantiations and inferences of the model.

\subsection{Scenario 1}

Bob is an expert fisherman. He is planning a fishing trip next Tuesday. The weather forecast shows that the visibility distance will be very low, at about 1.5 miles. He asks for a map that meets his needs and requirements.

Table 5 summarizes the concepts and role assertions that model the first scenario. On the one hand, knowing that the user has a "high" expertise, the reasoner classifies Bob as a professional user. Bob is engaged in activity $a$, an instance of the fishing class. This activity, being a subclass of context information has a context. The context of this activity is represented with instance $c 1$. Based on the context formalization (see Section 4.2), the system classifies $c 1$ as a ProfessionalFishingContext. On the other hand, the physical environment $e$ of the activity has VisibilityDistance $(v d)$ of about 1.5 miles. The visibility distance, as a dynamic context information, implies a situation $s$. Once again, the reasoner infers the class of the situation based on the situation formalization presented in Section 4.2. The inferred BadVisibility situation generates a context $c 2$. Then, the system classifies $c 2$ as an instance of BadVisibilityContext. The object properties hasActor, isTheContextOf, causedBy, generatedBy are inferred as inverse properties of engagedIn, hasContext, implies and generates, respectively. Each context is related to a set of thematic layers as follows (Listing 2-3): 
Listing 2: Thematic layers associated to ProfessionalFishingContext.

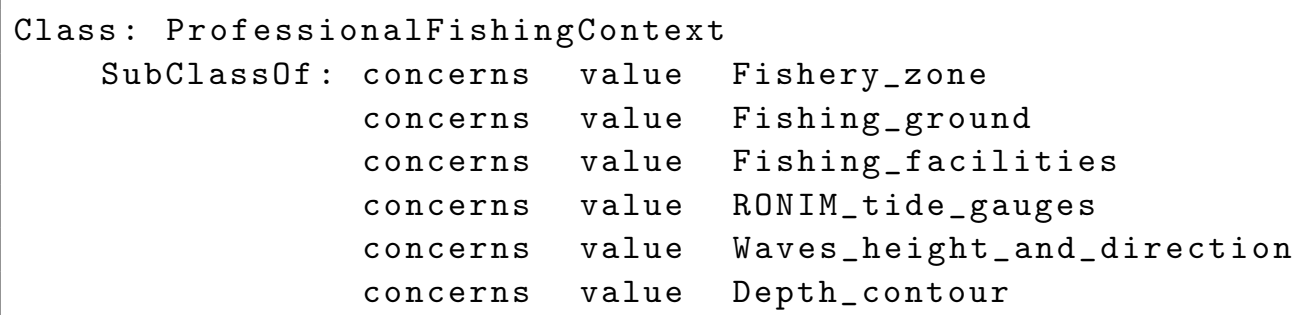

Listing 3: Thematic layers associated to BadVisibilityContext.

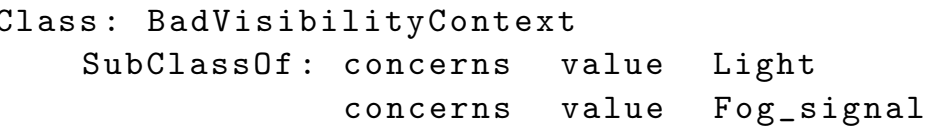

Table 5. Model instantiation by assertion and reasoning. Last column indicates the origin of the inference.

\begin{tabular}{|c|c|c|c|}
\hline Concepts and Roles & Asserted & Inferred & $\begin{array}{l}\text { Inference } \\
\text { Explanation }\end{array}$ \\
\hline User $(B o b)$ & $\checkmark$ & & \\
\hline hasExpertise (Bob, high) & $\checkmark$ & & \\
\hline ProfessionalUser $(B o b)$ & & $\checkmark$ & (9) \\
\hline Fishing $(a)$ & $\checkmark$ & & \\
\hline engagedIn $(B o b, a)$ & $\checkmark$ & & \\
\hline TemporalEntity $(t)$ & $\checkmark$ & & \\
\hline Instant (i) & $\checkmark$ & & \\
\hline hasBegining $(t, i)$ & $\checkmark$ & & \\
\hline inTemporalPosition ( $i$, Tuesday) & $\checkmark$ & & \\
\hline hasTime $(a, t)$ & $\checkmark$ & & \\
\hline hasLocation $(a, l)$ & $\checkmark$ & & \\
\hline Feature $(l)$ & $\checkmark$ & & \\
\hline hasGeometry $(l, g)$ & $\checkmark$ & & \\
\hline Context $(c 1)$ & $\checkmark$ & & \\
\hline hasContext $(a, c 1)$ & $\checkmark$ & & \\
\hline isTheContextOf $(c 1, a)$ & & $\checkmark$ & hasContext $^{-1}$ \\
\hline hasActor $(a, B o b)$ & & $\checkmark$ & engagedIn $^{-1}$ \\
\hline ProfessionalFishingContext $(c 1)$ & & $\checkmark$ & (16) \\
\hline Resources (layer1) & $\checkmark$ & & \\
\hline concerns (c1, layer1) & $\checkmark$ & & \\
\hline maybeInterestedIn (Bob, layer 1$)$ & & $\checkmark$ & Rule 1 \\
\hline PhysicalEnvironment $(e)$ & $\checkmark$ & & \\
\hline hasEnvironment $(a, e)$ & $\checkmark$ & & \\
\hline VisibilityDistance $(v d)$ & $\checkmark$ & & \\
\hline hasVisibilityDistance ( $v d, 1.5)$ & $\checkmark$ & & \\
\hline hasConditions $(e, v d)$ & $\checkmark$ & & \\
\hline Situation $(s)$ & $\checkmark$ & & \\
\hline implies $(v d, s)$ & $\checkmark$ & & \\
\hline causedBy $(s, v d)$ & & $\checkmark$ & implies $^{-1}$ \\
\hline BadVisibility (s) & & $\checkmark$ & (7) \\
\hline Context $(c 2)$ & $\checkmark$ & & \\
\hline generates $(s, c 2)$ & $\checkmark$ & & \\
\hline generatedBy $(c 2, s)$ & & $\checkmark$ & generates $^{-1}$ \\
\hline BadVisibilityContext (c2) & & $\checkmark$ & (8) \\
\hline Resources (layer2) & $\checkmark$ & & \\
\hline concerns $(c 2$, layer 2$)$ & $\checkmark$ & & \\
\hline maybeInterestedIn (Bob, layer 2$)$ & & $\checkmark$ & Rule 3 \\
\hline
\end{tabular}


In Table 5, layer1 and layer2 are instances of the resources class, and represent the set of thematic layers related to ProfessionalFishingContext and BadVisibilityContext, respectively. Once the appropriate contexts are deduced, the rule-based reasoning is applied to recommend the relevant thematic layers related to the contexts of use in which the user is involved. In the first scenario, Rules 1 and 3 infer the object property maybeInterestedIn between $\mathrm{Bob}$ and the thematic layers related to the inferred contexts of use (Figure 6).

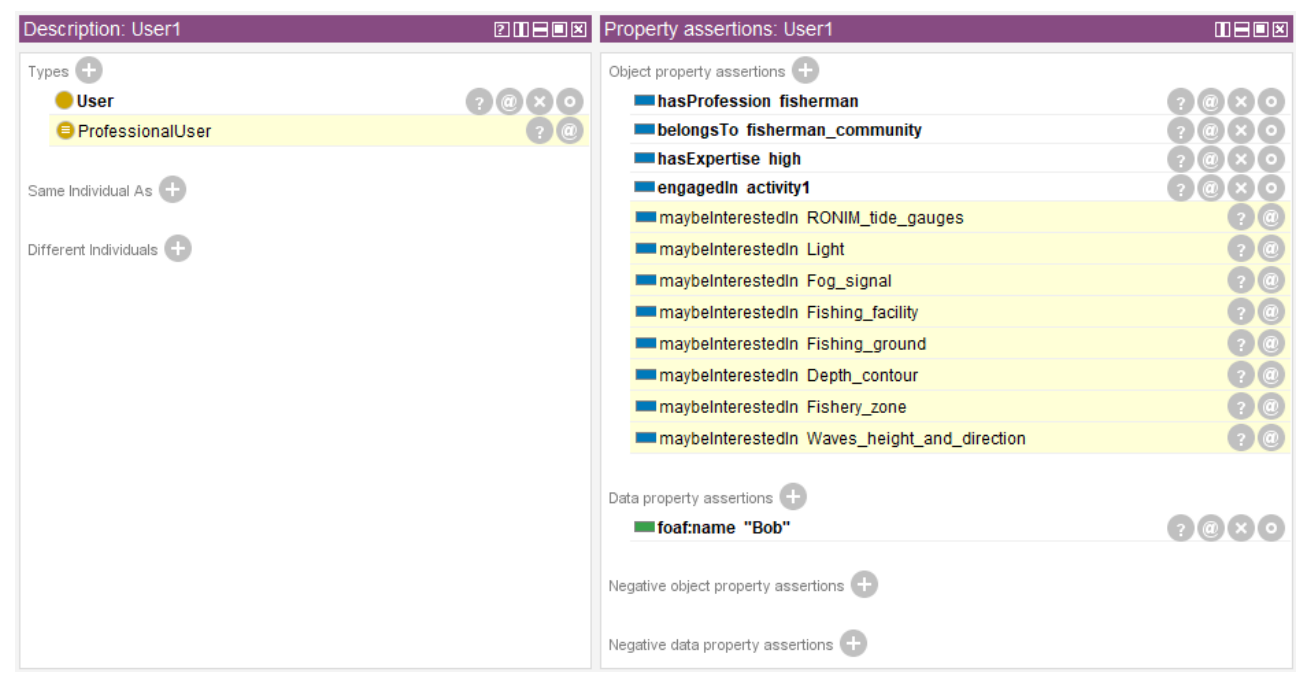

Figure 6. Results of inferences for scenario 1 using Protégé. Recommendation of thematic layers for Bob's on-demand map.

\subsection{Scenario 2}

Alice is a German tourist. She plans to rent a sailing boat with her friends during their holidays. She is an average sailor. She plans to sail from Jersey to Guernsey on the night of 8-9 November 2021. She is therefore looking for a map to guide her on her journey.

Table 6 shows the instantiation of the second scenario. Alice is engaged in a sailing activity with a medium expertise. Using ontological reasoning, the system classifies the context related to this activity as a SailingContext. The navigation context refers to the basic map layers/entities that help the traveler to navigate in normal conditions, such as weather, currents, tide, signals, beacons or guidance equipment (Figure 7). The activity takes place at night. This temporal dimension implies a situation. Based on the definitions of the situations, the reasoner classifies it as a BadVisibilitySituation. Sailing in a bad visibility situation requires additional layers concerning lighting or radar beacons (e.g., light or fog_signal layers in Figure 7). Thus, a second context is inferred: BadVisibilityContext. In this scenario, Rules 1 and 2 infer the recommendations to the cartographer. Figure 7 presents the result of the reasoning process and the set of thematic layers recommended for the user's on-demand map. 


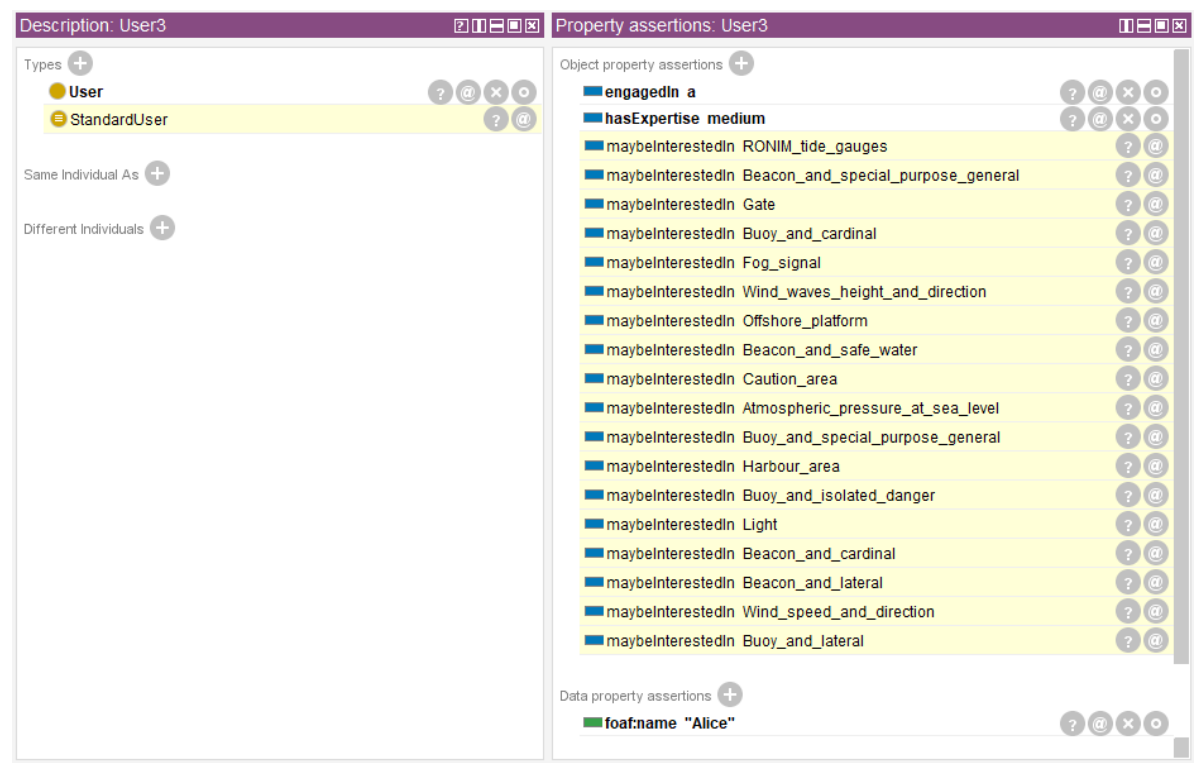

Figure 7. Results of inferences for Scenario 2 using Protégé. Recommendation of thematic layers for Alice's on-demand map.

Table 6. Scenario 2 instantiation by assertion and reasoning. Last column indicates the origin of the inference.

\begin{tabular}{|c|c|c|c|}
\hline Concepts and Roles & Asserted & Inferred & $\begin{array}{c}\text { Inference } \\
\text { Explanation }\end{array}$ \\
\hline User (Alice) & $\checkmark$ & & \\
\hline hasExpertise (Alice, medium) & $\checkmark$ & & \\
\hline StandardUser (Alice) & & $\checkmark$ & $(10)$ \\
\hline Sailing $(a)$ & $\checkmark$ & & \\
\hline engagedIn (Alice, $a)$ & $\checkmark$ & & \\
\hline hasLocation $(a, l)$ & $\checkmark$ & & \\
\hline Feature $(l)$ & $\checkmark$ & & \\
\hline hasGeometry $(l, g)$ & $\checkmark$ & & \\
\hline Context $(c 1)$ & $\checkmark$ & & \\
\hline hasContext $(a, c 1)$ & $\checkmark$ & & \\
\hline SailingContext (c1) & & $\checkmark$ & $(20)$ \\
\hline hasActor (a, Alice) & & $\checkmark$ & engagedIn $^{-1}$ \\
\hline isTheContextOf $(c 1, a)$ & & $\checkmark$ & hasContext $^{-1}$ \\
\hline Resource s(layer1) & $\checkmark$ & & \\
\hline concerns $(c 1$, layer 1$)$ & $\checkmark$ & & \\
\hline maybeInterestedIn (Alice, layer 1 ) & & $\checkmark$ & Rule 1 \\
\hline NightTime $(\mathrm{t})$ & $\checkmark$ & & \\
\hline hasTime $(a, t)$ & $\checkmark$ & & \\
\hline Situation $(s)$ & $\checkmark$ & & \\
\hline implies $(t, s)$ & $\checkmark$ & & \\
\hline causedBy $(s, t)$ & & $\checkmark$ & implies $^{-1}$ \\
\hline BadVisibility (s) & & $\checkmark$ & $(7)$ \\
\hline Context $(c 2)$ & $\checkmark$ & & \\
\hline generates $(s, c 2)$ & $\checkmark$ & & \\
\hline generatedBy $(c 2, s)$ & & $\checkmark$ & generates $^{-1}$ \\
\hline BadVisibilityContext (c2) & & $\checkmark$ & (8) \\
\hline Resources (layer2) & $\checkmark$ & & \\
\hline concerns $(c 2$, layer 2$)$ & $\checkmark$ & & \\
\hline maybeInterestedIn (Alice, layer 2 ) & & $\checkmark$ & Rule 3 \\
\hline
\end{tabular}

\section{Discussion and Conclusions}

In this paper, we present a knowledge-based recommendation approach for an ondemand mapping system. We address the first step of an on-demand mapping process 
by recommending to a cartographer the appropriate thematic layers, according to the user's requirements and context of use. For this, we propose a context modeling approach for contextual cartography based on a high-level ontology, taking into account different context dimensions (e.g., user, activity, time, location, environment, event, situation, and policy). Each high-level concept may be extended to a set of low-level concepts describing a context in a specific domain of application. For the purposes of this paper, we limit our case studies to maritime maps and, therefore, detail the low-level concepts involved, but the approach can be derived to other types of maps.

The knowledge-based recommendation approach relies on an ontological reasoning principle. Two types of reasoning are used to infer the knowledge of interest for ondemand maps: axiomatic reasoning and rule-based reasoning. The former infers the context(s) from contextual information, while the latter infers the relevant thematic layers based on the inferred context(s). In order to demonstrate the usability of the approach, we dealt with a particular domain: nautical maps. Some concepts related to the maritime domain were formalized in description logic for the axioms and in SWRL for the rules. The recommendation process was applied on two different scenarios. Although experts in mapping and knowledge engineering are needed to represent the application domain and define a set of contexts, the knowledge-based approach assures the cartographer of the quality of the recommendations through a reasoning process that matches the user's requirements to the relevant thematic layers. Currently, our work was carried out in consultation with a few cartographers and maritime navigation experts. Although the recommendations are in line with their expected results, further usability studies need to be carried out on a wider panel of cartographers and end-users to confirm the effectiveness of the recommendation process.

The recommendation process of our approach could be enhanced by going beyond the single recommendation of thematic layers presented in this paper. As a first way, we can recommend to the cartographer not only some thematic layers, but also the cartographic entities of interest, specific to each recommended layer. For example, if a user is involved in a navigation context where the boycar layer (i.e., cardinal buoys) is recommended to him or her, then depending on his/her location (spatial dimension), the system can recommend the set of cardinal buoys that exist in the practice area where he/she is planning his/her activity. In the same way, the system can recommend entities, taking into account the temporal dimension. For example, in a tourism context, the system can recommend cultural sites that are open during the user's activity.

Another way to be explored is to introduce a serendipity aspect in the recommendation process. Serendipitous recommendations would present some relevant, novel and unexpected thematic layers for the user. Unlike the proposed approach, where recommendations are derived from knowledge internal to the system (i.e., stored in the knowledge base), here, we are looking for recommendations derived from knowledge external to the system, such as Wikipedia categories, Wordnet or DBpedia. The main idea is to explore new recommendations having strong semantic links with the user's needs and that may be of interest. For instance, a standard user requesting an on-demand map in a fishing context may be recommended to have the wreckage sites. Indeed, the system, having determined a fishing context, could infer an interest in diving, as the two activities have a strong semantic relationship. Then, by analyzing the subcategories of diving in the Wikipedia categories, the system could finally recommend the diving sites or wreckage layers. On the one hand, this layer could be rather relevant and unexpected for a user, but on the other hand, it could reduce the quality or security of the recommendation, which may be important criteria for some applications. As a result, depending on the context of use, we have to weight the recommendation results between serendipitous recommendations (e.g., tourism context) and safe recommendations (e.g., navigation context). 
Author Contributions: Conceptualization, Bilal Koteich, Éric Saux and Wissame Laddada; methodology, Bilal Koteich and Éric Saux; validation, Bilal Koteich, Éric Saux and Wissame Laddada; formal analysis, Bilal Koteich, Éric Saux and Wissame Laddada; investigation, Bilal Koteich and Éric Saux; writing—original draft preparation, Bilal Koteich; writing—review and editing, Bilal Koteich, Éric Saux and Wissame Laddada. All authors have read and agreed to the published version of the manuscript.

Funding: Not applicable.

Institutional Review Board Statement: Not applicable.

Informed Consent Statement: Not applicable.

Data Availability Statement: Not applicable.

Conflicts of Interest: The authors declare no conflict of interest.

\section{References}

1. Cecconi, A.; Weibel, R.; Barrault, M. Improving Automated Generalisation for On-Demand Web Mapping by Multiscale Databases; Advances in Spatial Data Handling; Richardson, D.E., van Oosterom, P., Eds.; Springer: Berlin/Heidelberg, Germany, 2002; pp. 515-531.

2. Balley, S.; Regnauld, N. Models and standards for on-demand mapping. In Proceedings of the 25th International Cartographic Conference, Paris, France, 3-8 July 2011.

3. Cecconi, A. Integration of Cartographic Generalization and Multi-Scale Databases for Enhanced Web Mapping. Ph.D. Thesis, ETH Zurich, Zürich, Switzerland, 2003. [CrossRef]

4. Sarjakoski, T.; Sarjakoski, L.T. Chapter 7-A Real-Time Generalisation and Map Adaptation Approach for Location-Based Services. In Generalisation of Geographic Information; Mackaness, W.A., Ruas, A., Sarjakoski, L.T., Eds.; Elsevier: Amsterdam, The Netherlands, 2007; pp. 137-159. [CrossRef]

5. Bucher, B.; Buard, E.; Jolivet, L.; Ruas, A. The Need for Web Legend Services. Web and Wireless Geographical Information Systems. In Proceedings of the 7th International Symposium (W2GIS 2007), Cardiff, UK, 28-29 November 2007; Volume 4857, pp. 44-60. [CrossRef]

6. Foerster, T. Web-Based Architecture for On-Demand Maps-Integrating Meaningful Generalization Processing. Ph.D. Thesis, University of Twente, Enschede, The Netherlands, 2010.

7. Gould, N.M. Formalising Cartographic Generalisation Knowledge in an Ontology to Support On-Demand Mapping. Ph.D. Thesis, Manchester Metropolitan University, Manchester, UK, 2014.

8. Balley, S.; Baella, B.; Christophe, S.; Pla, M.; Regnauld, N.; Stoter, J. Map Specifications and User Requirements. In Abstracting Geographic Information in a Data Rich World: Methodologies and Applications of Map Generalisation; Burghardt, D., Duchêne, C., Mackaness, W., Eds.; Springer: Cham, Switzerland, 2014; pp. 17-52. [CrossRef]

9. Dey, A.K. Understanding and Using Context. Pers. Ubiquitous Comput. J. 2001, 5, 4-7. [CrossRef]

10. Abowd, G.D.; Dey, A.K.; Brown, P.J.; Davies, N.; Smith, M.; Steggles, P. Towards a Better Understanding of Context and ContextAwareness; Handheld and Ubiquitous Computing; Gellersen, H.W., Ed.; Springer: Berlin/Heidelberg, Germany, 1999 ; pp. $304-307$.

11. Chen, G.; Kotz, D. A Survey of Context-Aware Mobile Computing Research; Technical Report; Dartmouth College: Hanover, NH, USA, 2000.

12. Cabrera, O.; Franch, X.; Marco, J. A Context Ontology for Service Provisioning and Consumption. In Proceedings of the IEEE Eighth International Conference on Research Challenges in Information Science, Marrakesh, Morocco, 28-30 May 2014. [CrossRef]

13. Strang, T.; Linnhoff-Popien, C. A Context Modeling Survey. Workshop on Advanced Context Modelling, Reasoning and Management. In Proceedings of the Sixth International Conference on Ubiquitous Computing (UbiComp 2004), Nottingham, UK, 7-10 September 2004.

14. Wang, X.H.; Zhang, D.Q.; Gu, T.; Pung, H.K. Ontology based context modeling and reasoning using OWL. In Proceedings of the Second IEEE Annual Conference on Pervasive Computing and Communications Workshops, Orlando, FL, USA, 14-17 March 2004; pp. 18-22. [CrossRef]

15. Reichenbacher, T. Adaptive concepts for a mobile cartography. J. Geogr. Sci. 2001, 11, 43-53. [CrossRef]

16. Zipf, A. User-Adaptive Maps for Location-Based Services (LBS) for Tourism, Innsbruck. In Proceedings of the International Congress on Tourism and Communications Technologies in Tourism, Innsbruck, Austria, 17 May 2002. [CrossRef]

17. Nivala, A.; Sarjakoski, L.T. Need for Context-Aware Topographic Maps in Mobile Devices. In Proceedings of the 9th Scandinavian Research Conference on Geographical Information Science (ScanGIS'2003), Espoo, Finland, 4-6 June 2003; pp. 15-29.

18. Schilit, B.; Theimer, M. Disseminating active map informations to mobile hosts. Inst. Electr. Electron. Eng. Netw. 1994, 5, $2232-2242$. [CrossRef] 
19. Felfernig, A.; Jeran, M.; Ninaus, G.; Reinfrank, F.; Reiterer, S.; Stettinger, M. Basic Approaches in Recommendation Systems. In Recommendation Systems in Software Engineering; Robillard, M.P., Maalej, W., Walker, R.J., Zimmermann, T., Eds.; Springer: Berlin/Heidelberg, Germany, 2014; pp. 15-37. [CrossRef]

20. Ramezani, M.; Bergman, L.; Thompson, R.; Burke, R.; Mobasher, B. Selecting and Applying Recommendation Technology. In Proceedings of the International Workshop on Recommendation and Collaboration in Conjunction with International ACM Conference on Intelligent User Interfaces, IUI, Canaria, Spain, 13-16 January 2008; pp. 613-620.

21. Zanker, M.; Jessenitschnig, M.; Jannach, D.; Gordea, S. Comparing Recommendation Strategies in a Commercial Context. IEEE Intell. Syst. 2007, 22, 69-73. [CrossRef]

22. Pathak, B.; Garfinkel, R.; Gopal, R.D.; Venkatesan, R.; Yin, F. Empirical Analysis of the Impact of Recommender Systems on Sales. J. Manag. Inf. Syst. 2010, 27, 159-188. [CrossRef]

23. Force d'Action Navale. Directives Générales pour la Conduite Nautique (DG NAUT); Marine Nationale: Toulon, France, 2005.

24. Hourdry, D. Météorologie Maritime; Marine Nationale: Brest, France, 2018.

25. World Meteorological Organization (WMO). Guide to Marine Meteorological Services, 2018th ed.; World Meteorological Organization (WMO): Geneva, Switzerland, 2018.

26. Baader, F.; Calvanese, D.; McGuinness, D.L.; Nardi, D.; Patel-Schneider, P.F. (Eds.) The Description Logic Handbook: Theory, Implementation, and Applications; Cambridge University Press: Cambridge, MA, USA, 2003.

27. Le Guyader, D. Modélisation des Activités Humaines en mer Côtière. Ph.D. Theses, Université de Bretagne Occidentale, Brest, France, 2012.

28. Hobbs, J.; Pan, F. An ontology of time for the Semantic Web. ACM Trans. Asian Lang. Inf. Process. 2004, 3, 66-85. [CrossRef]

29. Tsatcha, D. Contribution à l'Extraction et à la Représentation des Connaissances de l'Environnement Maritime: Proposition d'une Architecture Dédiée aux Applications de Navigation. Ph.D. Thesis, Université de Bretagne Occidentale, Brest, France, 2014.

30. Gatin, G.; De Montaignac de Chauvance, H. Contribution à la Mise en Place d'un Système de Visualisation de Cartes Personnalisées; Technical Report; Institut de Recherche de l'Ecole Navale: Lanvéoc, France, 2020. 